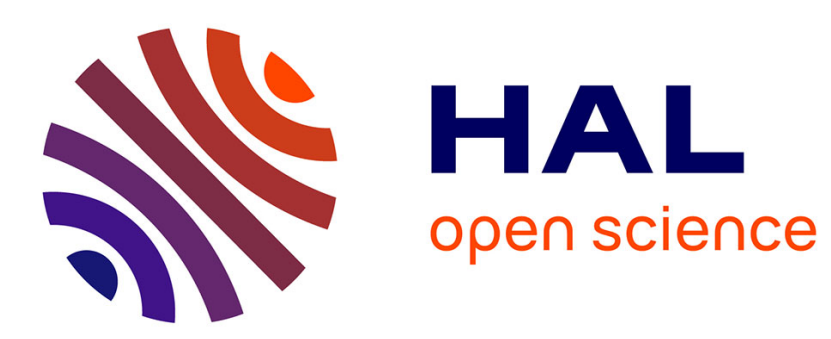

\title{
Delay range stability of a class of distributed time delay systems
}

Frédéric Gouaisbaut, Yassine Ariba

\section{To cite this version:}

Frédéric Gouaisbaut, Yassine Ariba. Delay range stability of a class of distributed time delay systems. Systems and Control Letters, 2011, 60 (3), pp.211-217. 10.1016/j.sysconle.2010.12.005 . hal01229611

\section{HAL Id: hal-01229611 \\ https://hal.science/hal-01229611}

Submitted on 17 Nov 2015

HAL is a multi-disciplinary open access archive for the deposit and dissemination of scientific research documents, whether they are published or not. The documents may come from teaching and research institutions in France or abroad, or from public or private research centers.
L'archive ouverte pluridisciplinaire HAL, est destinée au dépôt et à la diffusion de documents scientifiques de niveau recherche, publiés ou non, émanant des établissements d'enseignement et de recherche français ou étrangers, des laboratoires publics ou privés. 


\title{
Delay range stability of a class of distributed time delay systems
}

\author{
F. Gouaisbaut ${ }^{a}$ Y. Ariba ${ }^{b}$ \\ ${ }^{\mathrm{a}}$ CNRS; LAAS; 7, avenue du Colonel Roche, F-3107ry Toulouse, France. \\ Université de Toulouse; UPS, INSA, INP, ISAE ; LAAS ; F-31077 Toulouse, \\ France. \\ fgouaisb@laas.fr \\ ${ }^{\mathrm{b}}$ department of electrical engineering and computer science, Icam, 75 avenue de \\ Grande Bretagne - 31300 Toulouse, France. \\ yassine.ariba@icam.fr
}

\begin{abstract}
This paper is dedicated to the stability analysis of a class of uncertain distributed delay systems, the kernel of which can be modeled as a polynomial function of the delay. The results are constructed by rewriting the system as an uncertain interconnected model. Appropriate robust control tools, i.e. quadratic separation, are then used to address the stability issue. To this end, some relations that highlight relevant characteristics of the delayed term are added to the interconnected model leading then to the conservatism reduction. Finally, numerical examples show the effectiveness of the proposed method.
\end{abstract}

Key words: Stability, distributed delays, quadratic separation

\section{Introduction}

For several decades, the problem of time delay system stability has received a lot of attention (see the monographs by $[11,20]$ and references therein) since many dynamical processes can be described by functional differential equations. Distributed delay systems constitute a particular case of such systems, modelling cumulative effect of the past values of the dynamic. Practical issues of such models are numerous in the literature. Indeed, distributed delay systems are often used to model the time lag phenomenon in thermodynamics $[5,24]$ ), in ecology as well as epidemiology like predator-prey systems [8]. In all these cases, contrary to a discrete delay system, the use of a distributed 
kernel allows a thinner modelling of the interactions between the different system components. Nevertheless, establishing structural properties such as stability, in the presence of a distributed kernel, are much more difficult than the point wise delay case. A first possibility is to use frequency approaches like [17] or [2], but these techniques are restricted to nominal systems without uncertainties. A second popular way to establish robust stability remains the Lyapunov-Krasovskii method. Pioneer works [15] have proposed appropriate Lyapunov-Krasovskii functionals adding extra terms to the classical one to cope with the distributed delay term. It leads generally to Ricatti equations and the results reveal themselves very conservative due to the constrained choice of the Lyapunov-Krasovskii functional. These techniques have been enhanced, firstly by [6,23], and its descriptor model, and then recently refined by $[12,3]$ using either different bounding techniques (Moon or Jensen inequalities) or some slack variables [16]. Nevertheless, all these results entail an inherent conservatism due to the conservative choice of the Lyapunov-Krasovskii functional and are often restricted to a constant distributed kernel. An alternative method proposed by $\mathrm{Gu}$ et al [10] and [11], is to combine a very general class of Lyapunov Krasovskii functional and a discretization scheme to obtain numerically tractable stability conditions. This technique dealing with piecewise constant delay kernel gives a drastic reduction of the conservatism at the expense of the numerical burden. Another interesting approach proposed by $[19,18]$, dealing with a rational delay kernel, still relies on the use of a Lyapunov-Krasovskii approach and is combined with a full block S procedure to cope with the induced parametrized LMI. However, stability is still assessed only for a prescribed delay which does not provide robust criteria with respect to the delay.

In this paper, we adopt a different point of view to study the stability of such systems. First of all, we suppose that the distributed kernel is a polynomial function, a wider class of system compared to those generally studied in the literature. Furthermore, we propose to use the quadratic separation approach. Coming from robust control theory, such tools study the robust stability of a linear transformation equation interconnected with an uncertain matrix. This methodology has shown its efficiency in establishing some stability conditions for linear time delay systems with a constant delay [21,9] or time varying one [1]. Following the methodology proposed in [21], we model the distributed delay system as a linear equation connected to a set of operators composed of the integrator and extra operators related to the delayed dynamic. Then, in order to reduce the conservatism, some relations between the uncertain operators and higher successive derivatives of the state are exploited to derive new delay interval stability conditions. This new interesting result allow us to consider the stability of distributed delay system that may be unstable if the delay is set to zero.

The paper is organized as follows. Next section is devoted to some preliminaries about the distributed delay system and quadratic separation. Then section 2 formulates a first way to describe the distributed delay system as an inter- 
connected uncertain system. Section 4 gives the central results of the paper. Section 5 presents some numerical simulations.

Notations : The set $\mathbb{L}_{2 e}^{n}$ is the extended set of $\mathbb{L}_{2}^{n}$ which consists of all measurable functions $f: \mathbb{R}^{+} \rightarrow \mathbb{C}^{n}$ such that the following norm $\|f\|_{2}=$ $\left(\int_{0}^{\infty}\left(f^{*}(t) f(t)\right)\right)^{1 / 2} d t<\infty .1_{\mathrm{p}}$ represents the $p \times p$ identity matrix. $0_{\mathrm{p} \times \mathrm{q}}$ stands for the $p \times q$ zero matrix. $\operatorname{diag}(A, B)$ stands for the block diagonal matrix $\operatorname{diag}(A, B)=\left[\begin{array}{ll}A & 0 \\ 0 & B\end{array}\right] \cdot C^{+}$is the set of all points in the complex plane whose real part is positive.

\section{Problem statement}

We consider a distributed delay system of the form:

$$
\left\{\begin{array}{l}
\dot{x}(t)=A x(t)+\int_{-h}^{0}\left(A_{d}(\theta)+R(\theta)\right) x(t+\theta) d \theta \\
x(t)=\phi(t), \forall t \in\left[-h_{\max }, 0\right]
\end{array}\right.
$$

where $x(t) \in \mathbb{R}^{n}$ denotes the instantaneous state, the scalar $h>0$ is unknown and belongs to the interval $\left[h_{\min }, h_{\max }\right], A \in \mathbb{R}^{n \times n}$. The initial conditions $\phi$ for system (1) is a continuously differentiable function on $\left[-h_{\max }, 0\right]$. The function $A_{d}+R$ represents the distributed kernel of the delay system. The first part $A_{d}$ is supposed to be a polynomial function of $\theta$ and can thus be written as $A_{d}(\theta)=\sum_{i=0}^{r} A_{d i} \theta^{i}$, where $r$ is a positive integer corresponding to the polynomial order and $A_{d i} \in \mathbb{R}^{n \times n}, \forall i \in\{0, \ldots, r\}$ are real constant matrices. The second part $R$ represents a norm-bounded uncertainty structured as :

$$
R(\theta)=E \Delta(\theta) F
$$

where $E \in \mathbb{R}^{n \times n_{u}}, F \in \mathbb{R}^{n_{u} \times n}$ are constant known matrices and $\Delta \in \mathbb{R}^{n_{u} \times n_{u}}$ embeds the uncertain nature of $R$ and satisfies the inequality

$$
\Delta(\theta)^{*} \Delta(\theta) \leq 1_{\mathrm{n}_{u}} .
$$

The distributed delay system (1) can be reformulated as:

$$
\dot{x}(t)=A x(t)+\sum_{i=0}^{r} A_{d i} \int_{-h}^{0} \theta^{i} x(t+\theta) d \theta+E \int_{-h}^{0} \Delta(\theta) F x(t+\theta) d \theta .
$$

Remark 1 Taking the distributed kernel as a polynomial function is not so restrictive since from the Weierstrass approximation theorem [14], every suf- 
ficiently smooth function on a closed and compact interval $[-h, 0]$ can be approximated as closely as desired by a polynomial of a high enough order.

In this paper, we aim at finding LMI conditions which ensure the stability of (2) for all delays in an interval $\left[h_{\min }, h_{\max }\right]$ where $h_{\min }$ is possibly set to zero if delay dependent stability tests are looked for. To this end, we propose to use the quadratic separation framework developed by [13] and extended to the singular case by [21]. Well-posedness of feedback systems and its corrolary the quadratic separation is a fundamental concept for stability analysis of nonlinear and uncertain systems. It states that internal signals of a multivariable feedback connection of two systems $F$ and $G$ are unique and bounded under external disturbances if and only if the graph of $F$ is topologically separated from the inverse graph of $G$. Let consider the feedback connection depicted in Figure 1, where $\mathcal{E}$ and $\mathcal{A}$ are two possibly non-square matrices and $\nabla$ is an uncertain constant complex valued matrix of appropriate dimensions, that belongs to a given set $\mathbb{W}$. The stability definition can be expressed as follows:

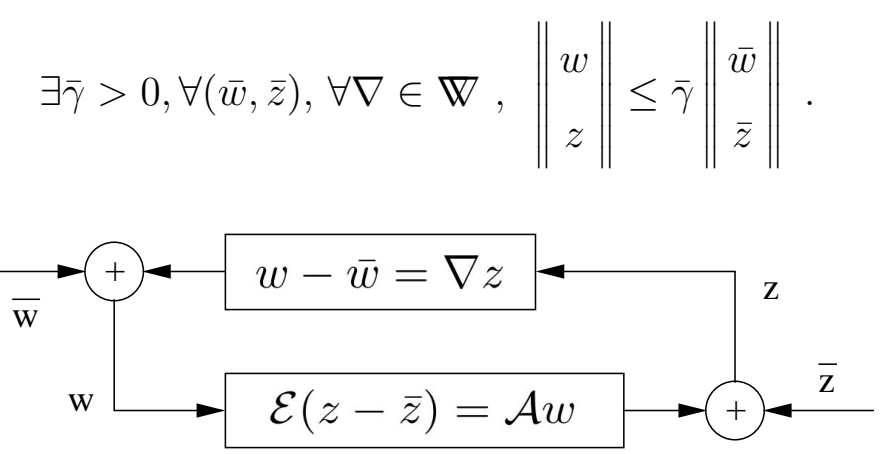

Fig. 1. Feedback system

The following theorem provides a condition for the well-posedness of such an interconnection, as well as its stability.

Theorem 1 ([21]) The uncertain feedback system of Figure 1 is well-posed and stable if and only if there exists a Hermitian matrix $\Theta=\Theta^{*}$ satisfying both conditions

$$
\begin{gathered}
{[\mathcal{E}-\mathcal{A}]^{\perp *} \Theta[\mathcal{E}-\mathcal{A}]^{\perp}>0} \\
{\left[\begin{array}{c}
I \\
\nabla
\end{array}\right]^{*} \Theta\left[\begin{array}{c}
I \\
\nabla
\end{array}\right] \leq 0 \quad, \quad \forall \nabla \in \mathbb{W} .}
\end{gathered}
$$

Remark that for simplicity of calculus, the last theorem is expressed for a full column rank matrix $\mathcal{E}$ which will be the case for all results presented in this paper. The proposed methodology can then be summarized as follows:

(a) Rewrite the original delay system (2) as an interconnected feedback system by choosing an appropriate set of operators. It generally includes the 
integral and delay related operators.

(b) Once the set $\mathbb{W}$ is properly defined, we look for a separator $\Theta$, which satisfies inequality (4) for all admissible uncertainties. The second inequality (4) is then fulfilled by construction.

(c) The first inequality (3) gives the stability condition to be tested and should be formulated as an LMI.

\section{$3 \quad$ Preliminary results}

Define the integral operator $\mathcal{I}$ and the distributed delay operators $\delta_{i}, \forall i \in$ $\{1, \ldots, r\}$, by

$$
\begin{aligned}
\mathcal{I}: \mathbb{L}_{2 e} & \rightarrow \mathbb{L}_{2 e}, \\
x(t) & \mapsto \int_{0}^{t} x(\theta) d \theta, \\
\delta_{i}: \mathbb{L}_{2 e} & \mapsto \mathbb{L}_{2 e}, \\
x(t) & \mapsto \int_{-h}^{0} \theta^{i} x(t+\theta) d \theta .
\end{aligned}
$$

Define also the uncertain operator $\nu$ by

$$
\begin{aligned}
\nu: \mathbb{L}_{2 e} & \rightarrow \mathbb{L}_{2 e}, \\
x(t) & \mapsto \int_{-h}^{0} \Delta(\theta) x(t+\theta) d \theta .
\end{aligned}
$$

Since $h$ is an unknown constant, the operators $\delta_{i}$ and $\nu$ can be conveniently expressed in the Laplace domain by $\delta_{i}(s)=\int_{-h}^{0} \theta^{i} e^{\theta s} d \theta$ and $\nu(s)=\int_{-h}^{0} \Delta(\theta) e^{\theta s} d \theta$. Following [13], we describe the uncertainty set $\mathbb{W}$ as:

$$
\begin{gathered}
\mathbb{W}=\left\{\nabla(s), \forall s \in \mathbb{C}^{+}\right\}, \\
\nabla(s)=\operatorname{diag}\left(s^{-1} 1_{\mathrm{n}}, \delta_{0}(s) 1_{\mathrm{n}}, \ldots, \delta_{r}(s) 1_{\mathrm{n}}, \nu(s)\right) .
\end{gathered}
$$

The definition of such an uncertainty set allows to describe the original distributed delay system (2) as a feedback interconnection as Figure 1 with

$$
\underbrace{\left[\begin{array}{c}
x(t) \\
\left(1_{\mathrm{n}} \delta_{0}\right)[x(t)] \\
\vdots \\
\left(1_{\mathrm{n}} \delta_{r}\right)[x(t)] \\
\nu[F x(t)]
\end{array}\right]}_{w(t)}=\underbrace{\left[\begin{array}{cccc}
\mathcal{I} 1_{\mathrm{n}} & & & 0 \\
& \delta_{0} 1_{\mathrm{n}} & & \\
& \ddots & \\
& & & \delta_{r} 1_{\mathrm{n}} \\
0 & & & \nu
\end{array}\right]}_{\nabla} \underbrace{\left[\begin{array}{c}
\dot{x}(t) \\
x(t) \\
\vdots \\
x(t) \\
F x(t)
\end{array}\right]}_{z(t)},
$$




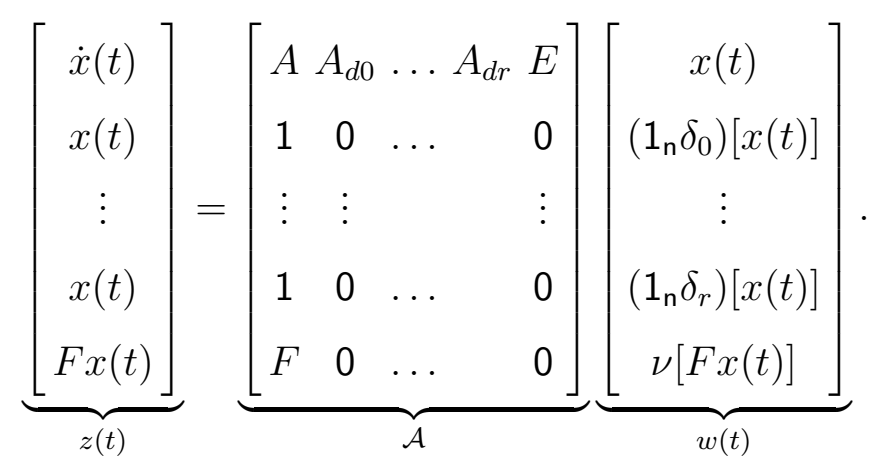

At this stage, we model the original system (2) as a feedback system described by a linear transformation (11) interconnected with an uncertain transformation $\nabla(s)$ belonging to a set $\mathbb{W}$ given by equation (9)-(10). In order to use Theorem 1, we construct a parametrized separator $\Theta$ satisfying the second inequality (4) as stated by the following lemma.

Lemma 1 Consider the uncertain operator $\nabla$ given by (9), a separator $\Theta$ satisfying (4) is given by

$$
\Theta=\left[\begin{array}{ll}
\Theta_{11} & \Theta_{12} \\
\Theta_{12}^{*} & \Theta_{22}
\end{array}\right]
$$

with

$$
\begin{aligned}
& \Theta_{11}=\operatorname{diag}\left(0_{n},-h_{\max }^{2} Q_{0}, \ldots,-\frac{h_{\max }^{2 r+2}}{(r+1)^{2}} Q_{r},-\alpha h_{\max }^{2} 1_{n_{u}}\right), \\
& \Theta_{12}=\operatorname{diag}\left(-P, 0_{n(r+1)+n_{u}}\right), \\
& \Theta_{22}=\operatorname{diag}\left(0, Q_{0}, \ldots, Q_{r}, \alpha 1_{n_{u}}\right),
\end{aligned}
$$

and $\alpha>0, P, Q_{0}, \ldots, Q_{r} \in \mathbb{R}^{n \times n}$ some positive definite matrices.

Proof 1 The key idea is to construct a separator for each uncertainty $s^{-1}$, $\delta_{0}, \ldots, \delta_{r}, \nu$ which composes $\nabla$ and then to concatenate all these relations to derive the whole separator $\Theta$. Noting that $\forall s \in \mathbb{C}^{+},\left|\delta_{i}(s)\right| \leq \frac{h_{\max }^{i+1}}{i+1}$, and following [9], a first set of separators for $\delta_{i}$, parametrized by $Q_{i}>0$ can be defined using the fact that

$$
\left[\begin{array}{c}
1_{\mathrm{n}} \\
\delta_{i} 1_{\mathrm{n}}
\end{array}\right]^{*}\left[\begin{array}{cc}
-\frac{h_{\max }^{2 i 2}}{(i+1)^{2}} Q_{i} & 0 \\
0 & Q_{i}
\end{array}\right]\left[\begin{array}{c}
1_{\mathrm{n}} \\
\delta_{i} 1_{\mathrm{n}}
\end{array}\right]<0
$$

A first set of separators for $\nu$, parametrized by $\alpha>0$ can be defined using the fact that

$$
\left[\begin{array}{c}
1_{\mathrm{n}_{u}} \\
\nu(s)
\end{array}\right]^{*}\left[\begin{array}{cr}
-\alpha h_{\max }^{2} & 0 \\
0 & \alpha
\end{array}\right]\left[\begin{array}{c}
1_{\mathrm{n}_{u}} \\
\nu(s)
\end{array}\right]<0 .
$$

At last, a separator for $\mathcal{I}$ is also parametrized by a positive definite matrix $P$ 
with:

$$
\left[\begin{array}{c}
1_{\mathrm{n}} \\
s^{-1} 1_{\mathrm{n}}
\end{array}\right]^{*}\left[\begin{array}{cc}
0 & -P \\
-P & 0
\end{array}\right]\left[\begin{array}{c}
1_{\mathrm{n}} \\
s^{-1} 1_{\mathrm{n}}
\end{array}\right]<0 .
$$

Consequently, gathering all these inequalities, the global separator associated to $\nabla$ defined by (10) can be chosen as (12).

Combining then Lemma 1 with Theorem 1, we propose the first delay dependent stability result :

Theorem 2 Given positive scalars $h_{\max }$ and $r$, if there exist positive definite matrices $P, Q_{i}$ for $i=\{0, \ldots, r\} \in \mathbb{R}^{n \times n}$, a positive scalar $\alpha$ such that the LMI condition (3) holds with $\Theta, \mathcal{E}$ and $\mathcal{A}$ defined as (12) and (11) then system (2) is asymptotically stable $\forall h \leq h_{\max }$.

Proof 2 In order to prove this theorem, we first model the original system (2) into an interconnected system (10)-(11). Then, applying Theorem 1, since Lemma 1 fulfils the second requirement (4), the first inequality (3) gives the condition to be tested.

\section{Main Results}

Now, we aim at introducing new relevant relations between the different components of the distributed delay system. The previous result can be improved by noting that given $i \geq 1$,

$$
\delta_{i}(s)=-\frac{(-h)^{i} e^{-s h}}{s}-\frac{i}{s} \delta_{i-1}(s) .
$$

This additional feature links the different operators each other: $\delta_{i}, \delta_{i-1}, s^{-1}$ and $e^{-h s}$, the delay operator defined by the

$$
\begin{aligned}
\mathcal{D}: \mathbb{L}_{2 e} & \rightarrow \mathbb{L}_{2 e}, \\
x(t) & \mapsto x(t-h) .
\end{aligned}
$$

Applying theses relations to the instantaneous state $x(t)$ give us a new set of relations between the state $x(t)$ and the delayed state $x(t-h)$ :

$$
\left\{\begin{array}{l}
\left(1_{\mathrm{n}} \delta_{0}\right)[\dot{x}(t)]=x(t)-x(t-h), \\
\left(1_{\mathrm{n}} \delta_{i}\right)[\dot{x}(t)]=-(-h)^{i} x(t-h)-i \delta_{i-1}[x(t)] .
\end{array}\right.
$$

As it appears that the derivative of $x(t)$ and $x(t-h)$ play a central role in these relations, we logically propose to consider the original model (2) as well 
as its derivative to obtain a new model:

$$
\left\{\begin{array}{c}
\dot{x}(t)=A x(t)+\sum_{i=0}^{r} A_{d i} \int_{-h}^{0} \theta^{i} x(t+\theta) d \theta++E \int_{-h}^{0} \Delta(\theta) F x(t+\theta) d \theta, \\
\ddot{x}(t)=A \dot{x}(t)+\sum_{i=0}^{r} A_{d i} \int_{-h}^{0} \theta^{i} \dot{x}(t+\theta) d \theta+E \int_{-h}^{0} \Delta(\theta) F \dot{x}(t+\theta) d \theta .
\end{array}\right.
$$

Obviously, if this delay system (18) is proved to be asymptotically stable, then the original delay system (2) will be also asymptotically stable. To take into account all these new relations (17) and (18), an extended uncertain transformation is required

$$
\mathbb{W}=\left\{\nabla(s), \forall s \in \mathbb{C}^{+}\right\},
$$

with

$$
\begin{aligned}
\nabla(s)=\operatorname{diag}( & 1_{2 \mathrm{n}} s^{-1}, 1_{\mathrm{n}} e^{-h s}, \\
& \left.1_{2 \mathrm{n}} \delta_{0}(s), \ldots, 1_{2 \mathrm{n}} \delta_{r}(s), 1_{2} \otimes \nu(s)\right) .
\end{aligned}
$$

Having chosen the complex matrix $\nabla$ (19) to model system (2), we now define the matrices as well as the internal signals which compose the linear transformation $\mathcal{E}(z-\bar{z})=\mathcal{A} \omega$ :

$$
z(t)=\left[\begin{array}{c}
\ddot{x}(t) \\
\dot{x}(t) \\
x(t) \\
\hline \dot{x}(t) \\
\frac{x(t)}{\vdots} \\
\hline \dot{x}(t) \\
x(t) \\
\hline F \dot{x}(t) \\
F x(t)
\end{array}\right], w(t)=\left[\begin{array}{c}
\dot{x}(t) \\
x(t) \\
\frac{x(t-h)}{\left(1_{\mathrm{n}} \delta_{0}\right)[\dot{x}(t)]} \\
\frac{\left(1_{\mathrm{n}} \delta_{0}\right)[x(t)]}{\vdots} \\
\frac{\left(1_{\mathrm{n}} \delta_{r}\right)[\dot{x}(t)]}{\left(1_{\mathrm{n}} \delta_{r}\right)[x(t)]} \\
\nu[F \dot{x}(t)] \\
\nu[F x(t)]
\end{array}\right] .
$$

Defining $\forall i \geq 0, \bar{A}_{d_{i}}=1_{2} \otimes A_{d_{i}}$, and $\bar{E}=1_{2} \otimes E$.

$$
\bar{A}=\left[\begin{array}{lll}
A & 0 & 0 \\
0 & A & 0
\end{array}\right], E_{1}=\left[\begin{array}{lll}
1 & 0 & 0 \\
0 & 1 & 0 \\
0 & 1 & 0 \\
0 & 0 & 1
\end{array}\right], V=\left[\begin{array}{l}
1 \\
\vdots \\
1
\end{array}\right] \in \mathbb{R}^{(r+2)}
$$


and

$$
\begin{gathered}
A_{1}=\left[\begin{array}{c}
V \otimes 1_{2 \mathrm{n}} \\
1_{2} \otimes \mathrm{F}
\end{array}\right], \\
A_{2}=\left[\begin{array}{ccccccccc}
0 & 1 & -1 & -1 & 0 & \ldots & \ldots & \ldots & 0 \\
0 & 0 & h 1 & 0 & -1 & -1 & 0 & \ldots & 0 \\
\vdots & \vdots & \vdots & \vdots & \ddots & \ddots & \ddots & \ddots & \vdots \\
0 & 0 & -(-h)^{r} 1 & 0 & \ldots & 0 & -r 1 & -1 & 0
\end{array}\right] .
\end{gathered}
$$

The matrices defining the linear transformation are then given by :

$$
\begin{aligned}
& \mathcal{E}=\left[\begin{array}{c}
E_{H} \\
0_{\mathrm{n}(\mathrm{r}+1) \times \mathrm{n}(2 \mathrm{r}+5)+2 \mathrm{n}_{\mathrm{u}}}
\end{array}\right], E_{H}=\operatorname{diag}\left(E_{1}, 1_{2 \mathrm{n}(\mathrm{r}+1)+2 \mathrm{n}_{\mathrm{u}}}\right), \\
& \mathcal{A}=\left[\begin{array}{cccc}
\bar{A} \bar{A}_{d 0} & \ldots \ldots \ldots \ldots \ldots \ldots & \bar{A}_{d r} & \bar{E} \\
\hline A_{1} & 0_{2 \mathrm{n}(\mathrm{r}+2)+2 \mathrm{n}_{\mathrm{u}} \times \mathrm{n}(2 \mathrm{r}+3)+2 \mathrm{n}_{\mathrm{u}}} \\
\hline A_{2} & 0_{\mathrm{n}(\mathrm{r}+1) \times 2 \mathrm{n}_{\mathrm{u}}}
\end{array}\right] .
\end{aligned}
$$

We now construct a separator $\Theta$ for the uncertain set described by (19).

Lemma 2 Consider the uncertain operator $\nabla$ given by (20), a separator $\Theta$ satisfying (4) is given by

$$
\Theta=\left[\begin{array}{cc}
\Theta_{11} & \Theta_{12} \\
\Theta_{12}^{*} & \Theta_{22}
\end{array}\right],
$$

where

$$
\begin{aligned}
& \Theta_{11}=\operatorname{diag}\left(0,-R,-h_{\max }^{2} Q_{0}, \ldots,-\frac{h_{\max }^{2 r+2}}{(r+1)^{2}} Q_{r},-h_{\max }^{2} \alpha_{1} 1_{\mathrm{n}_{u}},-h_{\max }^{2} \alpha_{2} 1_{\mathrm{n}_{\mathrm{u}}}\right), \\
& \Theta_{12}=\operatorname{diag}(-P, 0, \ldots, 0), \\
& \Theta_{22}=\operatorname{diag}\left(0, R, Q_{0}, \ldots, Q_{r}, \alpha_{1} 1_{\mathrm{n}_{\mathrm{u}}}, \alpha_{2} 1_{\mathrm{n}_{\mathrm{u}}}\right),
\end{aligned}
$$

with $P, Q_{0}, \ldots, Q_{r} \in \mathbb{R}^{2 n \times 2 n}, R \in \mathbb{R}^{n \times n}, r+3$ positive definite matrices and $\alpha_{1}, \alpha_{2}$ positive scalars.

Proof 3 This proof follows essentially the same line than the one of Lemma 1 , except that an additional uncertainty $e^{-h s}$, representing the point wise delay 
is added to $\nabla$. Noting that $\forall s \in \mathbb{C}^{+},\left|e^{-h s}\right| \leq 1$, we get

$$
\left[\begin{array}{c}
1_{\mathrm{n}} \\
e^{-h s} 1_{\mathrm{n}}
\end{array}\right]^{*}\left[\begin{array}{cc}
-R & 0 \\
0 & R
\end{array}\right]\left[\begin{array}{c}
1_{\mathrm{n}} \\
e^{-h s} 1_{\mathrm{n}}
\end{array}\right]<0 .
$$

Gathering the former relations (13), (14), it is straightforward to prove that separator (23) along with (20) satisfies inequality (4), which concludes the proof.

We are now ready to present a second original result.

Theorem 3 For given positive scalars $h, h_{\max }, r$ such that $0 \leq h \leq h_{\max }$, if there exists positive definite matrices $P, Q_{0}, \ldots, Q_{r} \in \mathbb{R}^{2 n \times 2 n}$ and $R \in \mathbb{R}^{n \times n}$, two positive scalars $\alpha_{1}, \alpha_{2}$ then system (2) is asymptotically stable for the given $h$, if the LMI condition (3) holds with $\Theta, \mathcal{E}$ and $\mathcal{A}$ defined as (23) and (22).

Proof 4 This proof is similar to the one of Theorem 2 and is thus omitted.

Remark 2 The proposed LMI condition relies on a matrix whose coefficients depend polynomially on the value of the delay $h$. Therefore, as $h$ belongs to a given interval $\left[h_{\min }, h_{\max }\right]$, some tools inherited from robust analysis can be used to transform the problem into a set of LMI which depend only on the values $h_{\min }$ and $h_{\max }$. In particular, two different techniques could be investigated:

- The proposed LMI is transformed into an LFT transformation depending on the variable $h$. This is always possible since $h$ appears rationnaly in the coefficients of the LMI. Then, a D-G scaling [22] can be used to caracterise $h$, as $h$ is a real variable belonging to an interval. Finally, the use of KYP lemma allows to derive an LMI, numerically tractable.

- Another interesting possibility relies on the use of Sum Of Squares methodology to developp an efficient criterion ([7] for a comprehensive treatment of such optimisation scheme).

Here, compared to these robust analysis tools, we propose an alternative method based on the introduction of extra variables and a complete rewriting of the original system. Indeed, we aim at expressing the relationship (17) as a set of equations, linear with respect to $h$ :

$$
\left\{\begin{array}{l}
\delta_{0}(s)=\frac{1-e^{-h s}}{s} \\
\delta_{1}(s)=\frac{h e^{-h s}}{s}-\frac{1}{s} \delta_{0}(s) \\
\delta_{i}(s)=-\left(h+\frac{i}{s}\right) \delta_{i-1}(s)-\frac{h(i-1)}{s} \delta_{i-2}(s), \forall i \geq 2 .
\end{array}\right.
$$

Furthermore, in order to take advantage from these new relations, following 
the work of [4], we consider the augmented vector:

$$
y(t)^{T}=\left[x^{(l-1) T}(t) x^{(l-2) T}(t) \ldots \dot{x}(t)^{T} x(t)^{T}\right] .
$$

This extended vector and its derivative are linked by the linear relation:

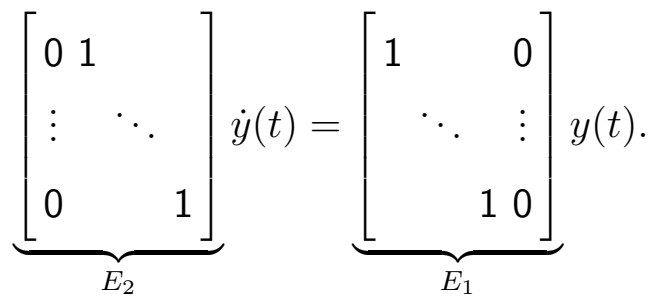

We now consider the following augmented system:

$$
\dot{y}(t)=\bar{A} y(t)+\sum_{i=0}^{r} \bar{A}_{d i} \int_{-h}^{0} \theta^{i} y(t+\theta)+\bar{E} \int_{-h}^{0} \bar{\Delta}(\theta) \bar{F} y(t+\theta),
$$

with $\bar{A}=1_{l} \otimes A, \bar{A}_{d i}=1_{l} \otimes A_{d i}, \forall i \in\{1, \ldots, l\}, \bar{E}=1_{l} \otimes E, \bar{F}=1_{l} \otimes F, \bar{\Delta}(\theta)=$ $1_{l} \otimes \Delta(\theta)$. In order to represent such a system into an interconnected feedback as Figure 1, we propose to use the following uncertain set:

$$
\mathbb{W}=\left\{\nabla(s), \forall s \in \mathbb{C}^{+}\right\}
$$

with

$$
\begin{aligned}
\nabla(s)=\operatorname{diag}( & 1_{\mathrm{n} \mid} s^{-1}, 1_{\mathrm{n} \mid} e^{-h s}, \\
& \left.1_{\mathrm{n} \mid} \delta_{0}(\mathrm{~s}), \ldots, 1_{\mathrm{n} \mid} \delta_{\mathrm{r}}(\mathrm{s}), 1_{\mathrm{l}} \otimes \nu(\mathrm{s})\right) .
\end{aligned}
$$

Choosing the uncertain transformation $\nabla$ as (28), the modelling of system (27) is described as follows. Firstly, we introduce the internal signals:

$$
z(t)=\left[\begin{array}{c}
\dot{y}(t) \\
y(t) \\
\hline y(t) \\
\vdots \\
y(t) \\
\bar{F} y(t)
\end{array}\right], w(t)=\left[\begin{array}{c}
y(t) \\
y(t-h) \\
\left(1_{n l} \delta_{0}\right)[y(t)] \\
\vdots \\
\left(1_{n l} \delta_{r}\right)[y(t)] \\
\left(1_{l} \otimes \nu\right)[\bar{F} y(t)]
\end{array}\right] .
$$

Then, we aim at describing system (27) using internal signals (30). 
- We first take into account the redundant equations in the internal signals and system equations (27):

$$
\underbrace{\left[\begin{array}{ccccc}
1 & 0 & 0 & \ldots & 0 \\
0 & 1 & 0 & \ldots & 0 \\
0 & 0 & 1 & \ldots & 0 \\
\vdots & \vdots & \ddots & \vdots \\
0 & 0 & 0 & \ldots & 1 \\
E_{2} & -E_{1} & 0 & \ldots & 0
\end{array}\right]}_{\mathcal{E}_{1}} z(t)=\underbrace{\left[\begin{array}{cccccc}
\bar{A} & 0 & \bar{A}_{d 0} & \ldots & \bar{A}_{d r} & \bar{E} \\
1 & 0 & 0 & 0 & \ldots & 0 \\
\vdots & 0 & 0 & 0 & \ldots & 0 \\
1 & 0 & 0 & 0 & \ldots & 0 \\
\bar{F} & 0 & 0 & 0 & \ldots & 0 \\
0 & 0 & 0 & 0 & \ldots & 0
\end{array}\right]}_{\mathcal{A}_{1}} w(t) .
$$

- Applying $E_{1} y(t)$ to relations (25) yields to the following equations:

$$
\left\{\begin{array}{l}
E_{1}\left(1_{n l} \delta_{1}\right)[y(t)]=E_{2} y(t)-E_{2} y(t-h), \\
E_{1}\left(1_{n l} \delta_{1}\right)[y(t)]=h E_{2} y(t-h)-E_{2}\left(1_{n l} \delta_{0}\right)[y(t)], \\
E_{1}\left(1_{n l} \delta_{i}\right)[y(t)]=-\left(h E_{1}+i E_{2}\right)\left(1_{n l} \delta_{i-1}\right)[y(t)]-h(i-1) E_{2}\left(1_{n l} \delta_{i-2}\right)[y(t)], \forall i \geq 2,
\end{array}\right.
$$

which can rewritten as linear relations using only $w(t)$ :

$$
\underbrace{\left[\begin{array}{ccccccc}
-E_{2} & E_{2} & E_{1} & 0 & 0 & 0 & \ldots 0 \\
0 & -h E_{2} & E_{2} & E_{1} & 0 & 0 & \ldots \\
0 & 0 & h E_{2}\left(h E_{1}+2 h E_{2}\right) & E_{1} & 0 & \ldots 0 \\
\vdots & \vdots & \ddots & \ddots & \ddots & \ldots & \ldots \\
0 & 0 & 0 & 0 & h(r-1) E_{2}\left(h E_{1}+r h E_{2}\right) & E_{1} & 0
\end{array}\right]}_{\mathcal{A}_{2}}
$$

Regrouping all the terms yields to a linear representation of the extended system:

$$
\underbrace{\left[\begin{array}{c}
\mathcal{E}_{1} \\
0
\end{array}\right]}_{\mathcal{E}} z(t)=\underbrace{\left[\begin{array}{c}
\mathcal{A}_{1} \\
A_{2}
\end{array}\right]}_{\mathcal{A}} w(t)
$$

Given the interconnected feedback given by equation (32) and (29), we propose a separator:

Lemma 3 Consider the uncertain operator $\mathbb{\mathbb { V }}$ given by (29), a separator $\Theta$ 
satisfying (4) is of the form (33)

$$
\Theta=\left[\begin{array}{cc}
\Theta_{11} & \Theta_{12} \\
\Theta_{12}^{*} & \Theta_{22}
\end{array}\right]
$$

where

$$
\begin{aligned}
& \Theta_{11}=\operatorname{diag}\left(0,-R,-h_{\max }^{2} Q_{0}, \ldots,-\frac{h_{\max }^{2 r+2}}{(r+1)^{2}} Q_{r},-h_{\max }^{2} \alpha_{1} 1_{\mathrm{n}_{u}}, \ldots,-h_{\max }^{2} \alpha_{l} 1_{\mathrm{n}_{\mathrm{u}}}\right), \\
& \Theta_{12}=\operatorname{diag}(-P, 0, \ldots, 0), \\
& \Theta_{22}=\operatorname{diag}\left(0, R, Q_{0}, \ldots, Q_{r}, \alpha_{1} 1_{\mathrm{n}_{\mathrm{u}}}, \ldots, \alpha_{l} 1_{\mathrm{n}_{\mathrm{u}}}\right),
\end{aligned}
$$

with $R, P \in \mathbb{R}^{n l \times n l}, Q_{0}, \ldots, Q_{r} \in \mathbb{R}^{n l \times n l}$ some positive definite matrices and $\alpha_{1}, \ldots, \alpha_{l} \in \mathbb{R}$ some positives scalars.

Proof 5 This proof is similar to the one of Lemma 1 and is thus omitted.

We are now in position to propose a delay-range stability criterion :

Theorem 4 For given positive scalars $h_{\min }, h_{\max }, r, l$ such that $h_{\min } \leq h_{\max }$, if there exists positive definite matrices $Q_{0}, \ldots, Q_{r} \in \mathbb{R}^{2 n(l+1) \times 2 n(l+1)}, P, R \in$ $\mathbb{R}^{n(l+1) \times n(l+1)}$ and a matrix $X \in \mathbb{R}^{2 n(l+1)(5+2 r) \times(n l(4 r+7)+n(3 r+5))}$, such that

$$
\left\{\begin{array}{l}
\Theta+X S\left(h_{\min }\right)+S\left(h_{\min }\right)^{T} X^{T}>0 \\
\Theta+X S\left(h_{\max }\right)+S\left(h_{\max }\right)^{T} X^{T}>0
\end{array}\right.
$$

with

$$
\begin{aligned}
S\left(h_{\min }\right) & =\left[\mathcal{E}-\mathcal{A}\left(h_{\min }\right)\right], \\
S\left(h_{\max }\right) & =\left[\mathcal{E}-\mathcal{A}\left(h_{\max }\right)\right],
\end{aligned}
$$

and $\Theta, \mathcal{E}, \mathcal{A}(h)$ defined as (33) and (32), then system (2) is asymptotically stable for $h$, such that $h_{\min } \leq h \leq h_{\max }$.

Proof 6 The proof follows the same lines than the one of Theorem 3. We use Theorem 2 to the uncertain interconnection defined by equations (29) and (32). By construction of the separator (33), the second requirement (4) is fulfilled. Then, the first requirement (3) gives that

$$
[\mathcal{E}-\mathcal{A}(h)]^{\perp *} \Theta[\mathcal{E}-\mathcal{A}(h)]^{\perp}>0
$$

This last inequality is not linear in $h$ but using Finsler Lemma, a sufficient condition for (35) is :

$$
\Theta+X[\mathcal{E}-\mathcal{A}(h)]+[\mathcal{E}-\mathcal{A}(h)]^{T} X^{T}>0,
$$


with $X$ a matrix of appropriate dimensions. This last inequality is then linear in $h$ and this inequality has to be assessed on the 2 vertices of the polytop generated by the interval on $h$, which concludes the proof.

Remark 3 (Robust Stability) System (2) is modeled in a robust framework, the extension of previous result to the robust case is straightforward and thus will not be explained.

Remark 4 (Conservatism reduction) As Theorem 3 is based on the results of Theorem 2, if we prove that system (1) is stable for $\left[0, h^{*}\right]$ with Theorem 2, it implies that the LMI of Theorem 3 is also fullfilled.

Remark 5 (Computationnal complexity) Numerically, the different LMI problems have the following characteristics:

- Theorem 2. The overall number of decision variables is $\frac{(r+2)(n+1) n}{2}+1$.

- Theorem 3. The overall number of decision variables is $(r+2)(2 n+1) n+$ $2+\frac{n(n+1)}{2}$.

- Theorem 4. The overall number of decision variables is $n(l+1)(r+1)(2 n(l+$ $1)+1)+n(l+1)(n(l+1)+1)+l+2 n^{2}(l+1)(5+2 r)(l(4 r+7)+3 r+5)$. These last comments on the numbers of variables for the LMI problem clearly indicate that even if one can prove some asymptotic property as l grows to infinity, it would be numerically intractable.

\section{$5 \quad$ Examples}

\section{Example 1 :}

Consider the following scalar example which has been considered in [19]:

$$
\dot{x}(t)=-0.5 x(t)+\int_{-h}^{0} \gamma(2,1,-\theta) x(t+\theta) d \theta
$$

with $x(t) \in \mathbb{R}, \gamma(k, \alpha, \theta)=\frac{\theta^{k-1} e^{-\theta / \alpha}}{\Gamma(k) \alpha^{k}}$ is the probability density function of the gamma distribution. Let choose a polynomial $P(\theta)$ and an uncertain set $\Delta(\theta)$ such that the function is entirely covered by $P \pm \delta$, where $\delta$ plays the role of the uncertainty. We construct the polynomial $P$ using a polynomial interpolation of order 4 in a least-squares sense. In this example $P(\theta)=-0.7466 \theta-0.644 \theta^{2}-$ $0.235 \theta^{3}-0.039 \theta^{4}-0.0026 \theta^{5}$, and we choose $\delta=0.05$. The results are illustrated in Figure 2. Using Theorem 3 and 4, we get then the results summarized in table 1.

\section{Example 2 :}




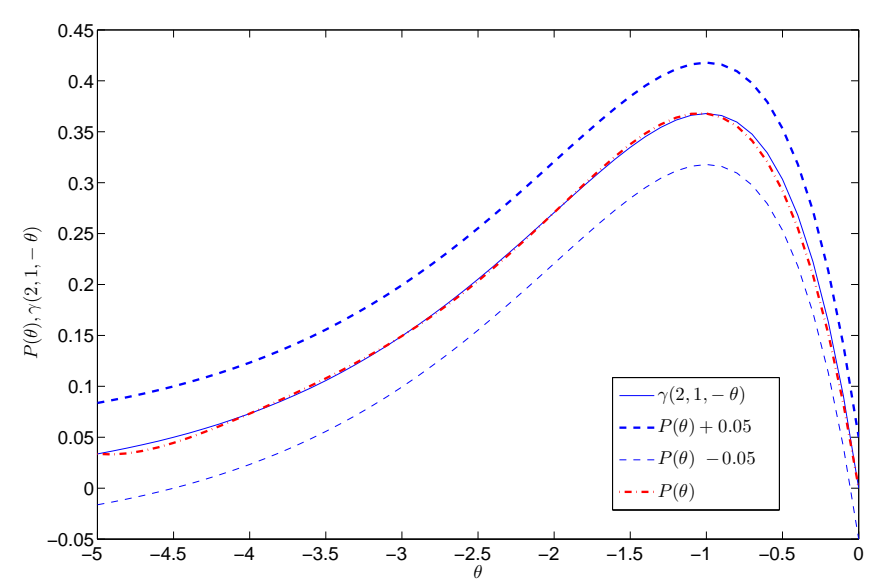

Fig. 2. $\gamma(\theta), P(\theta) \pm \delta$ for $\theta \in[-5 ; 0]$

Table 1

\begin{tabular}{|c|c|c|}
\hline Method & $h_{\max }$ & Numbers of variables \\
\hline Theorem 3, & 0.86 & 24 \\
Theorem $4, l=1$ & 1.480 & 2887 \\
Theorem $6,[19]$ & 1.442 & 14 \\
Analytical bound $[2]$ & 1.489 & - \\
\hline
\end{tabular}

Maximum allowable delay $h_{\max }$ for system (37)

Consider the distributed delay system:

$$
\dot{x}(t)=-2 x(t)+\int_{-h}^{0}\left(1+\theta+\theta^{3}\right) x(t+\theta) d \theta .
$$

Using an analytical method [2], system (38) is shown to be asymptotically stable for all delays less than 1.759. Using Theorem 3, we prove that system (38) is asymptotically stable $\forall h<1.0$. Then, choosing $h_{\text {min }}=0$ and using Theorem 4, we get the results summarized in table 2 .

Using an augmented vector to model the original system allows a reduction of conservatism. Nevertheless, for $l \geq 3$, increasing $l$ do not improve anything. Moreover, surprisingly, if the kernel $A_{d}(\theta)=1+\theta+\theta^{3}$ is modeled as a polynomial of order greater than $r$, taking the distributed kernel as $A_{d}(\theta)=\sum_{i=0}^{\mu} A_{d i}$ with $A_{d i}=0, \forall i \geq 3$, we introduce therefore fictitious operators $\delta_{r+1}, \delta_{r+2}, \ldots$, which slightly improved results as shown in Table 2. Combining these two effects, the choice of the polynomial degree and the number of higher derivatives, we expect an improvement of our results. Hence, using $r=9, l=9$, we prove that system is stable for $h \leq 1.758$, close to the analytical bound.

\section{Example 3 :}


Table 2

\begin{tabular}{|c|c|c|}
\hline Method & $h_{\max }$ & Numbers of variables \\
\hline Theorem $4, l=0$ & 1.32 & 322 \\
Theorem $4, l=1$ & 1.58 & 1499 \\
Theorem $4, l=2$ & 1.60 & 3530 \\
Theorem $4, l \geq 3$ & 1.61 & - \\
\hline \hline Theorem $4, l=0$ & $h_{\max }$ & Numbers of variables \\
\hline$r=3$ & 1.32 & 322 \\
$r=4$ & 1.43 & 459 \\
$r=5$ & 1.432 & 620 \\
\hline
\end{tabular}

Maximum allowable delay $h_{\max }$ for system (38)

This example is a second order distributed system of the form (2):

$$
\dot{x}(t)=\left[\begin{array}{cc}
0.2 & 0.01 \\
0 & -2
\end{array}\right] x(t)+\int_{-h}^{0}\left[\begin{array}{cc}
-1+0.3 \theta & 0.1 \\
0 & -0.1
\end{array}\right] x(t+\theta) d \theta
$$

Obviously this system is unstable for $h=0$. Furthermore, using the analytical method proposed by [2] and a gridding technique, this system appears to be stable for $0.195 \leq h \leq 1.71$. Using Theorem 4 over a sliding window, we obtain the following results, summarized in Table 3. Finally, gathering all

Table 3

\begin{tabular}{|c|c|c|c|}
\hline Theorem 4, & $h_{\mathrm{min}}$ & $h_{\max }$ & Numbers of variables \\
\hline$l=1, r=1$ & 0.23 & 1 & 2221 \\
$l=1, r=2$ & 0.21 & 1.2 & 3873 \\
$l=1, r=3$ & 0.2 & 1.29 & 5973 \\
\hline$l=2, r=1$ & 0.21 & 1 & 5240 \\
$l=2, r=2$ & 0.20 & 1.2 & 9134 \\
$l=2, r=3$ & 0.20 & 1.3 & 14034 \\
\hline
\end{tabular}

Intervall of stability $\left[h_{\min }, h_{\max }\right]$ for system (39) w.r.t. $l$ and $r$

these intervals, we prove then that system (39) is stable $\forall h \in[0.20,1.3]$. 


\section{Conclusion}

The paper is dedicated to the stability of distributed delay system by using the quadratic separation paradigm. It allows to consider to a wider class of distibuted kernel and to exploit some interactions between the different signals related to the delayed dynamics. Future works include extension of this work to more general kernels. Approximating the kernel by a polynomial and modelling the related error by a bounded uncertainty seems to be a promising way.

\section{References}

[1] Y. Ariba, F. Gouaisbaut, and D. Peaucelle. Stability analysis of time-varying delay systems in quadratic separation framework. In International conference on mathematical problems in engineering, aerospace and sciences (ICNPAA'08), Genoa, Italy, 2008.

[2] D. Breda, S. Maset, and R. Vermiglio. Pseudospectral differencing methods for characteristic roots of delay differential equations. SIAM Journal on Scientific Computing, 27(2):482-495, 2005.

[3] W.-H. Chen and W.X. Zheng. Delay-dependent robust stabilization for uncertain neutral systems with distributed delays. Automatica, 43(1):95 - 104, 2007.

[4] Y. Ebihara, D. Peaucelle, D. Arzelier, and T. Hagiwara. Robust performance analysis of linear time-invariant uncertain systems by taking higher-order timederivatives of the states. In $44^{\text {th }}$ IEEE Conference on Decision and Control and the European Control Conference (CDC-ECC'05), Seville, Spain, December 2005.

[5] Y. A. Fiagbedzi and A. E Pearson. A multistage reduction technique for feedback stabilizing distributed time-lag systems,. Automatica, 23(3):311-326, 1987.

[6] E. Fridman and U Shaked. A descriptor system approach to $\mathcal{H}_{\infty}$ control of linear time-delay systems. IEEE Transactions on Automatic Control, 47:253270, February 2002.

[7] Y. Genin, Y. Hachez, Yu. Nesterov, and P. Van Dooren. Optimization problems over positive pseudo-polynomial matrices. SIAM J. Matrix Anal. Appl., 25:5779, 2000.

[8] H. Gorecki, S. Fuksa, P. Grabowski, and A. Korytowski. Analysis and Synthesis of Time Delay Systems. John Wiley \& Sons, 1989.

[9] F. Gouaisbaut and D. Peaucelle. Robust stability of time-delay systems with interval delays. In $46^{\text {th }}$ IEEE Conference on Decision and Control (CDC'07), New Orleans, USA, December 2007. 
[10] K. Gu, Q.L. Han, A.C.J. Luo, and S.I. Niculescu. Discretized lyapunov functional for systems with distributed delay and piecewise constant coefficients. International Journal of Control, 74(7):737 - 744, 2001.

[11] K. Gu, V. L. Kharitonov, and J. Chen. Stability of Time-Delay Systems. Birkhäuser Boston, 2003. Control engineering.

[12] Q.-L. Han. A descriptor system approach to robust stability of uncertain neutral systems with discrete and distributed delays. Automatica, 40(10):1791 - 1796, 2004.

[13] T. Iwasaki and S. Hara. Well-posedness of feedback systems: insights into exact robustnessanalysis and approximate computations. IEEE Transactions on Automatic Control, 43:619-630, May 1998.

[14] H. Jeffreys and B. S. Jeffreys. Methods of Mathematical Physics. Cambridge University Press, 1988.

[15] V. B. Kolmanovskii and L. E. Shaikhet. Control of systems with aftereffect. American Mathematical Society, 1996.

[16] X.-G. Li and X.-J. Zhu. Stability analysis of neutral systems with distributed delays. Automatica, 44(8):2197 - 2201, 2008.

[17] C.-I. Morarescu, S.-I. Niculescu, and Gu K. Stability crossing curves of shifted gamma-distributed delay systems. SIAM Journal on Applied Dynamical Systems, 6(2):475-793, 2007.

[18] U. Munz, J. Rieber, and F. Allgower. Topics in Time-Delay Systems: Analysis, Algorithms, and Control, volume 388 of LNCIS, chapter Robust Stabilization and H-infinity Control of Uncertain Distributed Delay Systems, pages 221-231. Springer, Berlin, 2009.

[19] U. Munz, J. M. Rieber, and F. Allgower. Robust stability of distributed delay systems. In 17th World IFAC Congress, Seoul, Korea, July 2008.

[20] S.I. Niculescu. Delay Effects on Stability. A Robust Control Approach, volume 269 of Lecture Notes in Control and Information Sciences. Springer-Verlag, Heildelberg, 2001.

[21] D. Peaucelle, D. Arzelier, D. Henrion, and F. Gouaisbaut. Quadratic separation for feedback connection of an uncertain matrix and an implicit linear transformation. Automatica, 43(5):795-804, 2007.

[22] C.W. Scherer. Lmi relaxations in robust control. European Journal of Control, 12:3-29, 2006.

[23] L. Xie, E. Fridman, and U. Shaked. A robust $h_{\infty}$ control of distributed delay systems with application to combustion control. IEEE Transactions on Automatic Control, 46(12):1930-1935, 2001.

[24] F. Zheng and P.M. Frank. Robust control of uncertain distributed delay systems with application to the stabilization of combustion in rocket motor chambers. Automatica, 38(3):487 - 497, 2002. 